\title{
LA SÉMANTIQUE DU PROTOTYPE ET LES GENRES (LITTÉRAIRES)
}

\begin{abstract}
Wołowski Witold, La sémantique du prototype et les genres (littéraires) [The scmantics of prototype and (literary) genres]. Studia Romanica Posnaniensia, Adam Mickiewicz University Press, Poznań, vol. XXXIII : 2006, pp. 65-83. ISBN 83-232-1643-6, ISSN 0137-2475.
\end{abstract}

The proposal of this paper, situated at the meeting point of linguistics and poetics, is to discuss the applicabilty of same selected prototypical thesis to the research on litterary genres. It examines the following questions: status and internal structure of generic categorisations (hierarchical levels, graduality), J.-M. Adam's sequential theory, origin of prototypicality judgements in literature, archetypeprototype relation, difficulties in prototypical analysis of generic hybridity.

Il est difficile de prévoir exactement dans quelle direction vont s'engager dans les prochaines décennies les recherches sur la généricité discursive et littéraire, on peut pourtant affirmer qu'elles ont bifurqué depuis un bon moment. Dès lors, soit on choisit de rester fidèle à un structuralisme classique axé sur la morphologie de la matière, c'est-à-dire sur les effets proprement textuels et les configurations thématiques, soit on se laisse attirer par les approches pragmatico-cognitives visant à explorer le territoire intérieur et extérieur des actants de la communication : auteur, lecteur, spectateur, usager ou encore éditeur. Au moment où nous écrivons ces lignes, la mouvance psycho-socio-pragmatique exerce sans doute une attraction plus forte. En témoigne l'orientation d'un récent colloque sur la généricité où les mots clés ne s'énoncent pas essence, type, structure, classement, procédé ni même effet (terme beaucoup plus à la mode depuis l'article d'Adam et Heidemann 2004), mais perception, compétence, expérience, reconnaissance, mobilisation et même plaisir (cf. Macé 2005). Bref, l'accent a migré sur l'activité perceptive et cognitive des sujets ; la matière textuelle n'intéresse plus que sous une forme décomposée et recomposée par une conscience individuelle (l'imaginaire idiosyncrasique) ou collective (stéréotypes, prototypes).

1 Compétences, reconnaissance et pratiques génériques, Lausanne, 26-27.11.2004, Paris 2122.04.2005. 
Dans le paysage théorique ainsi dessiné - sans doute trop schématique, car plus que d'une polarisation il s'agit peut-être là d'optiques parallèles et en fin de compte complémentaires - il existe des théories qui occupent une position intermédiaire. Tel est le cas de la théorie des tropes qui nécessite une certaine prise en compte du pôle décodeur mais qui s'appuie sur le repérage d'indices présents dans le matériau verbal $^{2}$. Tel semble aussi le cas de la théorie du prototype (désormais notée TP) qui procède d'une réflexion générale sur la catégorisation et l'organisation de l'expérience chez le sujet percepteur, mais qui accorde en même temps une place importante à l'analyse des propriétés des objets linguistiques et de leurs référents conceptuels ou récls.

Le présent article propose une discussion sur cette dernière théorie et, en particulier, sur l'utilité, pour le domaine des genres littéraires, de quelques observations avancées dans le cadre des recherches sur le prototype.

Pour autant que nos connaissances permettent de le dire, on n'a pas souvent tenté de voir s'il est possible de traiter les faits de la généricité littéraire en termes de prototype, bien que les problèmes de catégorisation, qui se trouvent au centre des $\mathrm{TP}$, présentent en principe pour les études génériques un très vif intérêt. Pourtant, mis à part les travaux de linguistique textuelle d'Adam et certaines observations d'Amossy on n'entend pas encore beaucoup parler de prototype dans la poétique générique même si elle s'ouvre de plus en plus à cette approche comme le soulignent des travaux récents (Skibińska $2003: 73$ ).

\section{PROTOTYPE : ÉVOLUTION DU CONCEPT ${ }^{3}$}

«Sur quelles bases range-t-on telle ou telle chose particulière avec d'autres dans une même catégorie? " (Kleiber $1990: 13$ ). La TP s'organise autour de ce problème fondamental, cherchant à lui donner une nouvelle solution, solution qui permette notemment d'expliquer pourquoi on peut appeler $X$ ce qui ne semble pas être $\mathrm{X}$. Le projet ainsi conçu, on le voit bien, dépasse largement l'orbite de la sémantique lexicale à l'intérieur de laquelle il est développé. Ne peut-on pas dire en effet que la théorie des genres littéraires a exactement les mêmes préoccupations? En se posant des questions aussi essentielles, la TP ne dissimule pas ses ambitions universalistes : «Elle paraît - dit Kleiber - appropriée à tout phénomène impliquant

${ }^{2}$ Nous avons tenté, dans les travaux précédents, d'extrapoler cette théorie à l'étude des intérférences génériques: W. Wolowski, Vers une interprétation tropique de l'hybridité générique (à paraître).

${ }^{3}$ Les recherches sur les effets de prototypicalité ayant fait l'objet depuis le début des années soixante-dix d'une riche littérature, nous avons choisi de nous référer essentiellement à un seul ouvrage de synthèsc, La sémantique du prototype. Catégories et sens lexical de G. Kleiber (1990) qui fait intelligemment le point sur les remaniements subis par le prototype au cours d'une quinzaine d'annces de recherches. Cette synthèse, il faut le préciser, est rattachée à une conception restreinte du prototype que l'on peut opposer aux conceptions générales de la stéréotypie (Skibińska 2003 : 63). 
une catégorisation ». Le traitement prototypique s'étendrait ainsi sur les artefacts, le vocabulaire abstrait, les verbes, les prépositions et même sur les scripts et scénarios, unités complexes d'analyse conversationnelle (Kleiber $1990: 101-103)$ ). Avec les scripts et les scénarios - genres simples au sens bakhtinien du terme - la frontière des genres littéraires n'est pas encore franchie, mais celle des genres du discours si.

Essayant de saisir théoriquement les comportements langagiers dénaturés, si l'on peut dire ainsi, par l'usage ordinaire du langage, la théorie du prototype s'en prend surtout au modèle classique de catégorisation, celui des conditions nécessaires et suffisantes (CNS). Dans ce modèle, on le sait, la catégorisation s'effectue sur la base de propriétés communes : un objet fait partie d'une catégorie s'il vérifie un certain nombre d'attributs constituant le dénominateur commun de la catégorie". L'idée de prototype permettrait donc d'affaiblir cette contrainte.

Qu'est-ce donc que le prototype? La notion n'est pas une, elle s'affine en trois étapes. Dans un premier temps, le prototype est considéré comme «le meilleur exemplaire, (...) le meilleur représentant ou l'instance centrale d'une catégorie », ce à quoi s'ajoute une précision importante "(il) n'est vraiment considéré comme le meilleur exemplaire que s'il apparaît comme étant celui qui est le plus fréquemment donné comme tel » (Kleiber $1990: 48-49$ ). Avec le temps, le prototype devient une entité de plus en plus abstraite, une idée que l'on se fait de ce meilleur exemplaire, pour se fixer définitivement comme conglomérat de traits saillants (typiques d'une catégorie) dont le mode d'existence est totalement virtuel.

Sur le plan littéraire, ce principe ferait qu'au prototype du roman ou de la pièce de thêâtre pourrait ainsi ne correspondre aucune réalisation textuelle effective. Ici surgit une difficulté, car il semble inconcevable d'établir un jeu abstrait de caractéristiques (pertinentes pour un genre et choisies dans un ensemble raisonnable de celles-ci) qu'aucune cuvre n'incarne : c'est l'extrême diversité des réaliastions actualisant un genre qui rend inimaginable une telle situation. $\mathrm{Vu}$ le nombre de textes constituant le corpus littéraire mondial, l'opération même d'appariement avec un tel modèle serait difficile à effectuer.

La qualification de meilleur demande des explications. On se pose en effet spontanément la question de savoir pourquoi les sujets trouvent tel exemplaire meilleur que d'autres. La réponse est la suivante : «parce qu'il possède les propriétés considérées comme typiques de la catégorie». Mais alors «qu'est-ce qui fait d'une propriété une bonne propriété, une propriété typique de la catégorie» (Kleiber $1990: 61-62$ ) ? A cette question, il y a deux réponses : « les traits typiques seront les traits qui apparaissent le plus souvent pour les membres d'une catégorie ». A cette fréquence « ordinaire » s'ajoute une fréquence contrastive pour

${ }^{4}$ Le modèle se résume en trois propositions : 1) les concepts ou catégories sont des entités aux frontières clairement délimitées ; 2) un $X$ est $Y$ ou ne l'est pas, selon qu'il satisfait ou non aux conditions critériales de la catégorie $\mathrm{Y} ; 3$ ) les membres d'une même catégorie ont un statut catégoriel égal (Kleiber $1990: 22$ ). 
laquelle les chercheurs anglophones emploient le terme de cue validity ${ }^{5}$ Le trait à cue validity élevée est un trait particulièrement distinctif, doté d'une grande vertu discriminative, souvent présent chez les membres d'une catégorie et en même temps absent des autres catégories. Pour le mode dramatique, par exemple, le trait 'présence de la didascalie de la source locutoire' possède ainsi une cue validity bien supérieure au trait 'présence de répliques en style direct', ces demières pouvant se rencontrer aussi bien dans un récit. La cue validity de ce trait est même extrêmement faible dans la mesure où il entre sans conteste dans la composition du prototype du roman : on n'imagine pas un roman prototypique sans dialogues.

Ainsi défini, le prototype foumit dans la version standard de la théorie à la fois un principe de catégorisation et d'organisation des catégories, ce qui conduit à la formulation de six thèses dans lesquelles se condense toute la TP standard ${ }^{6}$ :

1: La catégoric a une structure interne prototypique ; 2 : Le degré de représentativité d'un exemplaire correspond à son degré d'appartenance à la catégorie ; 3 : Les frontières des catégories ou des concepts sont floues ; 4 : Les membres d'une catégoric ne prćsentent pas de propriétés communes à tous les membres; c'est une ressemblance de famille qui les regroupe ensemble ; 5 : l'appartenance à une catégorie s'effectue sur la base du degré de similarité avec le prototype; 6 : elle ne s'opère pas de façon analytique, mais de façon globale (Klcibcr $1990: 51$ ).

La seconde version de la TP marque une rupture. Elle résulte d'une « révision drastique » (Kleiber $1990: 149$ ) qui conduit à l'abandon de l'ensemble des thèses sous-tendant la version standard : de six il n'en subsiste qu'une seule... Des ruines de la version standard doit ainsi se lever la théorie étendue, "version multiréférentielle beaucoup plus puissante » (Kleiber 1990:146), capable de rendre compte des phénomènes de polysémie, impossibles à maîtriser dans la première version. Paradoxalement, l'accroissement de la puissance de la théorie entraîne un affaiblissement considérable de la notion de prototype qui «n'a plus le statut d'entité fondatrice de la structure catégorielle (...). Ayant plusieurs sources possibles, elle n'est plus considérée que comme un effet, ce qui amène (...) à parler de degrés de prototypicalité plutôt que de prototype » (Kleiber 1990:150). C'est dans le même sens qu'il faut lire cette observation de Klinkenberg: "Chaque classe a en effet un ou des prototypes, qui en sont le ou les meilleurs exemples » $(2000: 103)$. On finit aussi par conclure au caractère non-décisif du mécanisme d'appariement prototypique pour la catégorisation. Vu la disparition du prototype comme représentant des catégories et comme facteur de leur structuration, tout l'édifice tient sur l'affirmation suivante : "la relation qui unit les différents membres d'une même caté-

${ }^{5}$ Pour ce qui concerne la notion même de cue validity, il semble d'ailleurs qu'on puisse lui trouver un homologue dans le terme de phénotype utilisé dans les études génériques slaves pour désigner dans une ceuvre littéraire les traits qui «décident de sa non-ressemblance (et à la fois sa resscmblance) avec d'autres œuvres " (Głowiński et al., 2000).

${ }^{6}$ Pour les observations définitives concernant ces thèses, voir Conclusion. 
gorie, quelle que soit cette catégorie, est celle de resemblance de famille » (Kleiber $1990: 152$ ).

La notion de ressemblance de famille, fondatrice de tout une architecture théorique, mérite un bref examen. Le concept est communément associé au nom de Wittgenstein qui l'utilise pour faire percevoir des similitudes plus ou moins vagues entre différentes activités de jeu (échecs, tennis, comptines) : «on y voit un réseau complexe de similitudes qui se chevauchent et s'entrecroisent : il s'agit parfois de similitudes globales, parfois de similitudes de détail » $(1953: 31-32)$. Tout autre chose est la ressemblance de famille sous sa forme « théorisée », c'est-à-dire exprimée en formule mathématique prétendant à la scientificité. La formule de la catégorie basée sur l'air de famille s'énonce donc ainsi : $\mathrm{AB}, \mathrm{BC}, \mathrm{CD}, \mathrm{DE}$, etc. où les variables correspondent à des propriétés et les conjonctions binaires de propriétés aux objets rentrant dans la catégorie. Remarquons que de l'objet $\mathrm{AB}$ (possédant les traits $\mathrm{A}$ et $\mathrm{B}$ ) à l'objet $\mathrm{CD}$ (présentant des caractéristiques $\mathrm{C}$ et $\mathrm{D}$ ) disparaît toute propriété commune pouvant légitimer la co-catégorisation. En présence d'une thèse aussi lourde en conséquences, le lecteur est en droit d'attendre une exemplification qui se respecte. Or, cette attente restera à jamais frustrée. Il n'est pas étonnant dans ces conditions que le concept ait été vivement critiqué par certains spécialistes. Voici un commentaire très réservé de Ducrot ${ }^{7}$ :

La solution habituelle (...) consiste à recourir à une métaphorc introduite par Wittgenstein, celle de ressemblance de famille (la bienséance actuelle commande de se pâmer devant la profondeur de cette notion, et de l'utiliser de façon dogmatique, alors qu'elle a, dans le texte original, une fonction purcment critique). Mais il est bien difficile de dire en quoi consiste cet air de famille (...) On peut supposer qu'il s'agit d'un trait commun à tous (...). Mais cette conception de l'air de famille réintroduit l'idée d'une condition nécessaire et suffisante, contre laquelle s'est justement construitc la théorie dcs prototypes (1999 : 290-291).

Savoureuse ironie que celle de Ducrot qui rappelle la donnée basique de la nécessité d'un dénominateur commun pour établir une classe, et qui n'imagine absolument pas qu'on puisse en construire une sur la base de rien. C'est pourtant cela qu'admettaient explicitement les défenseurs de la TP. Lakoff fait dériver du concept de la ressemblance de famille l'idée «que les membres d'une catégorie peuvent être reliés les uns aux autres sans qu'ils aient une propriété en commun qui définisse la catégorie " (Kleiber $1990: 151$ ).

On comprend bien que l'application un peu cavalière du principe de la ressemblance de famille au domaine des genres littéraires donnerait feu vert à toutes les fantaisies. Relier par un air de famille générique un sonnet de Du Bellay, un roman de Perec et une pièce de Billetdoux ne représenterait dès lors aucune difficulté...

'Baylon et Mignot se montrent eux aussi très sceptiques : « La notion d'air de famille demcure intuitive, et en la réintroduisant comme on l'a fait sans pouvoir lui donner de contenu précis, on manque aux exigences scientifiques » (1995:133). 


\section{SENS LEXICAL ET STATUT DES CATÉGORIES GÉNÉRIQUES : HIÉRARCHISATION ET GRADUALITÉ}

Pour appliquer le modèle prototypique aux catégorisations littéraires, il est nécessaire d'opérer une généralisation", c'est-à-dire de passer de la sémantique, territoire originaire du prototype (version restreinte), à la poétique, terre d'adoption, et de voir dans quelle mesure la structure sémique et référentielle du langage est superposable à la structure du système générique. Le nombre d'analogies, on le devine, est important, mais il n'est pas illimité.

Réfléchissons donc un instant sur le statut des catégories génériques littéraires. Il est inutile de persuader qui que ce soit - c'est du moins notre opinion - du caractère "sémantique » des catégories génériques littéraires, quel qu'en soit le degré de généralité. Le genre tel qu'il est défini par la tradition, par l'état actuel de la recherche, tel qu'il est " prototypisé " par les sujets, constitue incontestablement une macrostructure sémantique, un grand signe, un ensemble de « sèmes » que l'on pourrait nommer - avec plus ou moins de bonheur - diégèmes, mimèmes, lyrèmes, etc. La poétique générique est ainsi une sénantique à l'échelle macro-structurale.

On doit pourtant faire face à cette première objection : l'œuvre littéraire est un objet sémiotique complexe (Schaeffer 1989:79) et cette complexité dépasse la seule dimension sémantique. Cela est vrai en un sens, mais il n'est pas faux non plus de soutenir que tous les aspects de l'acte discursif, à savoir les éléments du cadre communicationnel et ceux de la réalisation syntaxique ${ }^{9}$ sont réductibles aux effets de sens, c'est-à-dire, en fait, à la sémantique. Les genres littéraires constituant des combinaisons de traits formels ou thématiques, isolables au même titre que les unités componentielles d'un sémème, peuvent donc théoriquement être appréhendés à travers des théories relatives aux autres objets sémantiques. Voyons d'autres analogies.

Comme le système lexical d'une langue, le système des genres est organisé, sur le plan catégoriel, suivant deux axes, horizontal (structuration interne des catégories) et vertical (structuration intercatégorielle). Comme celle du système lexical, la catégorisation générique présente une structure hiérachique dans laquelle se combinent les deux dimensions susmentionnées, l'intra- et l'inter-catégorialité.

Pour ce qui est de la hiérarchisation taxinomique, Kleiber (1990) se concentre sur deux modèles, celui de Berlin et celui de Rosch. Le premier, qui prétend fournir le principe de base de toutes les classifications populaires, dégage cinq niveaux. Au lieu de reproduire à la lettre l'exemple de l'auteur (plante / arbre / chêne / chêne

${ }^{8}$ Cette généralisation n'est qu'un aspect d'une vaste opération de traduction des structures linguistiques en structures poétiques souhaitée déjà en 1967 par Greimas affirmant qu'« une même méthodologic de base peut servir à l'analyse des objets linguistiques et poétiques " (Revue internationale des sciences sociales, vol. XIX, 1, repris dans $1970: 271$ ).

${ }^{9}$ En présentant les conceptions de Granger, Mounin donne un titre significatif à un chapitre dc son ouvrage (1997/1972) La syntaxe est aussi une sémantigue (1997/1972 : 172). 
vert / chêne vert méditerranéen), nous remplissons le tableau (à titre d'essai) avec des noms de genres $^{10}$ :

$\begin{array}{lll}\text { Règne } & \text { Genre épique / Mode narratif } & \text { Genre dramatique / Mode dialogal } \\ \text { Forme de vie } & \text { récit } & \text { dialoguc } \\ \text { Genre } & \text { roman, conte de féc } & \text { pic̀ce de théâtre, dialogue philosophique } \\ \text { Espèce } & \text { roman policier } & \text { comédie } \\ \text { Variété } & \text { roman policier cn argot } & \text { farce de ménage }\end{array}$

Le second, celui de E. Rosch, nous ramène à trois niveaux :

$\begin{array}{lll}\text { Niveau superordonné } & \text { Mode narratif } & \text { Mode dialogal } \\ \text { Niveau basique } & \text { roman } & \text { tragédic } \\ \text { Niveau subordonné } & \text { roman policier } & \text { tragédic religieuse }\end{array}$

En construisant ces modèles, la TP poursuit un objectif précis : elle essaye de trouver le niveau de catégorisation privilégié par les usagers d'une langue (le plus économique sur le plan de l'informativité, le plus accessible pour la mémoire, etc.). Pour ce qui est du domaine littéraire, cette optique de recherche nous semble un peu moins intéressante, bien qu'il ne soit nullement interdit de se demander ce que répondrait instinctivement monsieur Untel interrogé dans le métro sur le genre du livre qu'il est en train de lire. Répondrait-il " un roman », " une pièce de théâtre ", en recourant au terme du niveau basique, ou préciserait-il tout de suite qu'il lit « un conte surréaliste " ? Il est douteux qu'il donne des précisions sans qu'on le lui demande explicitement. Il est aussi douteux qu'il réponde, sinon par une plaisanterie de connaisseur, qu'il lit de l'épique...

La question des modèles de hiérarchisation catégorielle nous fait en revanche prendre conscience d'un certain nombre d'autres problèmes. Le premier est celui de la différence fondamentale qui existe entre le système lexical et le système des genres. Elle concerne le rapport entre le niveau des catégories subordonnées et le niveau des instances individuelles. En effet, si la différence est négligeable entre un moineau prototypique et n'importe quel exemplaire particulier de moineau que nous voyons dans la rue, elle est énorme entre Polyeucte de Corneille et Le Comédien et la Grâce de Ghéon, toutes les deux œuvres exemplifiant l'espèce drame religieux. Cela rend pratiquement invalide en littérature toute conception qui postulerait d'instaurer en prototype une quelconque réalisation particulière. Le prototype du genre littéraire ne saurait être qu'abstrait et multiple dans ses instanciations.

Une autre observation importante qui s'impose lorsqu'on regarde les schémas de hiérarchisation est la suivante: le ou les niveaux superordonnées de la catégorisation littéraire tendent à être structurés par des oppositions de type et de mode, alors que les niveaux subordonnés tendent à faire intervenir des oppositions d'ordre

${ }^{10}$ Nous ne prenons ici en considération que les genres narratif et dramatique, étant donné que le genre dit lyrique ne correspond à aucune distinction modalc supplémentairc. Le lyrique est unc catégoric (au sens de Blanché) transversale, c'est-à-dire transcendant modes, types et genres. 
thématique. Au niveau superordonné, on oppose ainsi mode narratif et mode dialogal (ou discours monogéré vs pseudo-polygéré), le narratif et le dramatique se différenciant en effet par le type d'énonciation qui les caractérise, donc par un critère linguistique universel. Par contre, au niveau basique et aux niveaux subordonnés, nous avons affaire aux genres littéraires proprement dits, c'est-à-dire aux institutions consacrées par la tradition culturelle.

Remarquons enfin que lors du passage du modèle à 5 niveaux au modèle à 3 niveaux, les catégories génériques littéraires nous mettent dans un sérieux embarras. Tout d'abord, il importe de noter que le nombre de niveaux pourrait peut-être se réduire à deux ou augmenter jusqu'à $\mathrm{X}$ pour des catégories les plus riches en étages. Ensuite, il faut se demander sur quelle base ranger telle catégorie à tel niveau. Ceci nous amène à reposer l'éternel problème de la définition des catégories génériques correspondant aux dénominations léguées par la tradition. Ce problème est simple à résoudre, puisqu'il est de fait insoluble comme le montre magistralement Schaeffer. Dans $Q u$ 'est-ce qu'un genre littéraire (1989), s'interrogeant sur la spécificité de la dénomination générique, il remarque que les noms de genres désignent des référents définis par recours à des critères hétérogènes relevant des cinq aspects de l'acte discursif. $\mathrm{Ce}$ qui en résulte, c'est l'impossibilité de trouver un plan définitionnel commun sur lequel traiter sonnet, nouvelle et vaudeville. Ceci dit, on ne doit pourtant pas déclarer l'impossibilité de toute hiérarchisation catégorielle intelligente au sein du pseudo-système des genres. La configuration que nous venons de proposer pèche sans doute par des inexactitudes, mais semble résister - au moins grosso modo - à la critique.

Pour en revenir donc à notre modèle provisoire, il faut aussi observer que les dénominations comme récit ou - surtout - roman pour le genre épique, ainsi que celle de pièce de théâtre pour le genre dramatique, devraient être considérées comme termes relevant du niveau de base. Or, récit et roman, communément rangés au même niveau (avec conte, nouvelle etc.), appartiennent en fait à des niveaux différents (récit est un terme qui tend à être tenu pour plus général); de même, le terme de pièce de théâtre semble n'être qu'une sous-catégorie du terme dialogue, dans la mesure où ce dernier comprend également le dialogue philosophique à la Platon ou, plus littéraire, celui d'un Leopardi des Operette morali.

Mettant en évidence la hiérachisation catégorielle, la TP accentue également la gradualité des propriétés. On sait bien en effet que toute œuvre est $\grave{a}$ un certain degré conforme aux lois de son genre, au profil de sa catégorie. La manière scalaire d'appréhender les choses n'est pourtant pas toujours de règle. Mitterand semble ne pas s'y conformer lorsqu'il intitule un des chapitres de sa Littérature française du $X X^{e}$ siècle (1996) Inclassables: Giono, Gracq, Yourcenar. Il est clair qu'on peut proposer des catégorisations incongrues où il n'y ait pas de rubrique prévue pour certains objets, néanmoins le qualificateur inclassable n'a plus vraiment droit de cité dans une réflexion qui se veut respectueuse des acquis théoriques réalisés au 
cours du dernier quart du siècle : tout est désormais classable, il suffit seulement de s'entendre sur le degré d'appartenance de tel objet à une ou plusieurs classes.

Dans ce contexte, il faut reconnaître que le grand mérite des théories prototypiques est justement de mettre en valeur le caractère graduel des propriétés. Ducrot lui-même reconnaît que les recherches sur le prototype ont permis d'« introduire une sorte de gradualité dans des catégories (...) où les critères linguistiques habituels de la gradualité sont difficiles à utiliser " (Ducrot et Schaeffer 1999 : 289). Dès lors, on commence à parler de degré d'appartenance (à une catégorie), de degré de représentativité, de gradient de prototypie, etc. On attribue à tel objet non seulement une étiquette fixe qu'un spécialiste un peu pédant pourrait contester, mais aussi un degré, une gradience, un coefficient qui apportent une spécification plus difficilement contestable. Cette attitude semble avoir une grande importance pour les études littéraires qui sont elles aussi confrontées aux cas difficiles, discutables, marginaux.

La médaille a toutefois deux faces : d'un côté l'intervention de la saisie graduelle offre une prise sur l'intercatégoriel, l'inclassable, l'hybride, l'idiosyncrasique ; de l'autre elle semble conduire vers une impasse. En effet, s'il existe des domaines qui admettent naturellement les critères de gradualité (tout un chacun comprendra très bien ce qu'est une boisson alcoolisée à $5 \%$, à $15 \%$ et à $45 \% \ldots$, d'autres domaines paraissent plus résistants : dire à propos d'une cuvre littéraire qu'elle est un roman à $30 \%$ n'apprendra pas grand-chose même à un spécialiste. Et puis, si X est roman à $30 \%$, à quelle proportion d'autres genres participent-ils à la formation de l'ensemble ? Et à quoi aboutirait la comparaison du taux de "romanicité" d'un Père Goriot et d'une Eugénie Grandet ? 93\% contre 94\% ? On voit bien la fragilité de la démarche.

En outre, il semble que l'on s'intéresse peu, en littérature, aux explications chiffrées. Savoir que L'Interrogatoire de Pinget n'est roman qu'à $1 \%$, ou que l'Acte sans paroles 1 de Beckett est une pièce à $0,5 \%$, à quoi cela peut-il nous avancer au fond? A un tableau plein de chiffres bien ronds, on préférera toujours une phrase, même mal tournée, qui énonce une vérité, même para-scientifique. La valeur graduée en littérature, si elle veut susciter un intérêt, ne saurait donc être une valeur exprimée en chiffres, mais plutôt par recours aux quantificateurs linguistiques naturels. Quelle que soit la stratégie adoptée, l'important est en tout cas d'avoir la conscience d'un continuum des formes sur lequel plus d'une segmentation est opérable et dans lequel se laissent enregistrer différentes intensités.

\section{LE PROTOTYPE DANS L'ANALYSE TEXTUELLE}

Le prototype s'introduit dans l'analyse textuelle, littéraire et générique par les travaux de J.-M. Adam (1992-2001), promoteur de la théorie séquentielle de la textualité. Mais cette introduction ne se fait pas sans un léger glissement d'accent. 
Le changement d'optique qu'opère la théorie des séquences vient du fait que les efforts d'Adam ne se focalisent pas sur l'organisation prototypique de la perception des objets discursifs, ni sur la structuration prototypique des catégories typologiques, ni même sur l'application éventuelle de la notion d'air de famille aux catégorisations génériques; ils tendent à caractériser de manière complète cinq types de séquences que l'on rencontre, à proportions variables, dans différents genres du discours. Il n'y est pas question d'évaluation du degré de représentativité, ni de ségrégation des traits particulièrement saillants de telle catégorie de référents. L'objectif d'Adam est simplement de relever les caractères distinctifs de cinq types de séquences (narrative, descriptive, argumentative, explicative et dialogale) sans tenter d'ordonner ces caractères définitoires dans une perspective hiérarchique. Ce qu'Adam cherche à définir, c'est donc des types plutôt que des prototypes. En abordant la narration, il précise du reste lui-même qu'il s'agit pour lui de «définir ce qui fait la spécificité de ce type de mise en texte » (2001:45), encore qu'il parle aussi dans la même page de la recherche du «modèle de la séquence narrative de base ». "De base » est un élément important, puisqu'il n'y a pas d'équivalence totale entre propriétés spécifiques et basiques. Bien que la dernière formulation de l'objectif soit plus proche des préoccupations strictement prototypiques, Adam réalise surtout la première tâche, puisqu'il réunit les «critères pour une définition du récit " (1) succession d'événements;2) unité thématique; 3) des prédicats transformés ; 4) un procès ; 5) causalité narrative d'une mise en intrigue ; 6) une évaluation finale - $2001: 45-56$ ). Cette définition, riche en composantes, fournit ainsi un profil très complet du type et non du prototype du récit. On imagine en effet sans difficulté des récits prototypiques sans présence d'anachronies (critère 5) ou sans énoncés facilitant au lecteur la compréhension de la totalité de l'aventure narrée (critère 6). C'est du reste ce que semble confirmer en quelque sorte D. Combe dans un article peu cité aujourd'huj et pourtant très intéressant de 1989 où il propose une définition linguistique du récit :

Enoncé supérieur ou égal à la proposition dans une phrase, simple ou complexe, remplissant d'une manière dominante la fonction référentielle grâce à la modalité assertive, signifiant l'idée d'action ou d'événement chronologique et logique par l'intermédiaire d'un prédicat où l'idée de temps est impliquée, attribuée à un thème, dont le signifié est par là-même anthropomorphe (1989: 165).

En faisant abstraction justement des deux caractéristiques dont nous venons de signaler la non nécessité, la définition de Combe ressemble davantage à une saisie prototypique, même si, là encore, l'établissement du prototype n'entre pas vraiment dans l'intention de l'auteur.

On pourrait établir de manière semblable deux ou plusieurs définitions du texte dramatique lesquelles exigeraient la possession d'un nombre de traits variable. La définition minimale du texte dramatique peut se ramener, selon les conclusions auxquelles aboutissent nos recherches précédentes, à deux points : 1) altemance de 
tours de parole entre actants qui partagent le même espace-temps fictif et qui poursuivent un échange pouvant être qualifié d'interactif ; 2) la présence des répliques et de la didascalie de la source locutoire (DSL) qui les précède à chaque fois. Pour ce qui est de la théâtralité textuelle, nous ne voyons pas d'autres critères essentiels. Or, défini comme cela, le modèle ne saurait constituer le prototype du texte dramatique. En effet, dans la conception commune, le texte de théâtre fait apparaître outre la DSL, d'autres types de didascalie. De plus, il est normalement divisé en actes et en scènes, et il comporte une intrigue assez serrée qui avance vers un dénouement. Tel qu'il fonctionne dans l'imaginaire collectif, le texte théâtral possède donc plus de propriétés que n'en comporte la définition-noyau constituée de deux caractères nécessaires et suffisants. Le prototype est donc une représentation incomplète, schématique".

La démarche d'Adam, se plaçant dans une perspective prototypique par l'emploi du terme de prototype dans le titre de l'ouvrage, ne diffère donc guère de celles qui utilisent le modèle des CNS pour la constitution des catégories. Il y a même pire : Adam ne semble pas trop se soucier du caractère nécessaire ou suffisant des attributs critériaux qu'il relève: ils sont mis tous sur le même plan. C'est que le dessein d'Adam est d'aboutir à une typologie, donc à un système, même s'il ne s'agit, comme il l'annonce lui-même, que d'une typologie parmi d'autres $(2001: 11)$. Malgré la modestie et les réserves de l'auteur, il faut bien reconnaître cependant que le modèle séquentiel est très puissant et analytiquement productif, puisqu'il semble a priori possible de ramener tout texte, litteraire ou autre, à une combinaison des cinq types de séquence décrits dans ce modèle.

Un autre point réclame encore une brève analyse, c'est celui de la relation entre séquence (typique ou pseudo-prototypique) et genre. Cette relation n'est pas en effet clairement définie. A lire la remarque suivante, on pourrait presque croire qu'il s'agit d'un rapport de subordination : «La structure globale des textes ne s'explique pas totalement par les combinaisons séquentielles car elle est, avant tout, réglée à un niveau trés supérieur par les genres de discours" (Adam 2001 : 43). Parmi ces demiers, Adam cite sonnet et comédie. L'expression genres de discours comprend ainsi aussi les genres littéraires au sens le plus classique du terme. La forme de séquence paraît donc être en quelque sorte déterminée par le genre du discours, y compris le discours littéraire. Mais en réalité, ce rapport n'est pas celui de subordination. Séquence narrative est sans doute un terme plus général que roman ou même récit et séquence dialogale englobe outre le genre théâtral d'autres genres de l'écriture et la conversation ordinaire, ce qui lui confère une portée bien supérieure à celle du genre dramatique. Il n'empêche que, pour que la structure

${ }^{11}$ C'est dans ce sens précisément que Wierzbicka distingue propriétés essentielles et prototypiques. Les premières : "le plus petit ensemble de traits qui, pris ensemble, garantissent que tout objet qui les possède sera généralement reconu comme appartenant à la catégorie en question ", les secondes : typiques mais pas essentielles pour la conception du référent $(1985: 60)$. 
globale des textes particuliers puisse apparaître comme résultante des facteurs génériques et typologiques, il faudrait savoir distinguer les deux régimes, ce qui ne va pas toujours de soi.

Inspiré par les recherches en prototypie, le modèle séquentiel d'Adam présente donc avec le modèle prototypique stricte sensu un rapport relâché. En outre, tout en ayant partie liée avec la réflexion générique, la théorie séquentielle, d'orientation plus linguitique, se situe paradoxalement à un niveau inférieur (déterminé) et à la fois plus général (cinq types subsumant bien plus de genres) par rapport à celui du genre discursif (et littéraire) ${ }^{12}$. Si Adam rencontre sur son chemin les catégories génériques littéraires, c'est qu'elles interferent largement au niveau superordonné avec les catégories typologiques : l'épique et le dramatique, pour ne pas employer les étiquettes plus modernes, désignent à la fois des grands genres et des grands types. Lorsque Genette propose de désigner les genres narratif et dramatique par le terme linguistique de mode (1986:98), il exprime on ne peut mieux le sentiment de cette ambivalence : il sait parfaitement que les deux genres se différencient au fond par le mode d'énonciation, c'est-à-dire par un critère structural-pragmatique de caractère universel.

En dépit des écarts qui le séparent des grandes thèses de la TP, Adam n'en reste pas moins le premier adaptateur de cette théorie aux analyses génériques du discours littéraire. Quand il dit que les genres discursifs sont des "catégories prototypiques-stéréotypiques (...) définissables par des tendances ou des gradients de typicalité, par des faisceaux de régularités et des dominantes plutôt que par des critères trop stricts » (1999: 93-94), Adam se situe - au moins théoriquement - en pleine optique prototypique.

\section{JUGEMENTS DE PROTOTYPICALITÉ EN LITTÉRATURE}

Il serait absurde de nier le fait que le domaine littéraire ignorc les phénomènes qui se produisent dans tout l'univers de la signification : «les sciences sociales, les sciences du langage et les études littéraires reconnaissent que les phénomènes de stéréotypie sont inévitables ", observent Amossy et Herschberg Pierrot (1997: 118). Confronté à un texte littéraire, le lecteur procède en effet comme n'importe

${ }^{12}$ Le problème de la relation type-genre est traité dans quelques travaux récents, cf. Bcacco (2004) et Kerbrat-Orecchioni et Traverso (2004). Ces dernières, se référant aux travaux d'Adam, proposent de distinguer les G1 et $\mathrm{G} 2$ «deux types d'objets qui peuvent également prétendre au label des genres ». Les genres 1 se définissent comme « catégories de textes plus ou moins institutionnalisées dans une société donnée. Les genres 2 sont des types plus abstraits de discours caractérisés par certains traits de nature rhétorico-pragmatique, ou relevant de leur organisation discursive ». Beacco souligne également le caractère abstrait et théorique du type : «l'activité classificatoire des genres discursifs a ćté contaminée par la classification relative aux types de discours : celle-ci lui est parallèle, mais elle se fonde sur un autre matériau que les noms de genres, puisque les classes de types discursifs sont des entités abstraites, non médiatisées par le lexique d'une langue » (2004:112). 
quel usager du langage devant un message comportant une dimension générique, devant un genre de message. Il fait des hypothèses, des approximations, des rapprochements avec des modèles, des meilleures instances, des cas typiques ou des exemplaires connus. La démarche prototypique est un comportement des plus spontanés, sinon le plus spontané, et ceci n'est pas le fait des seuls prophanes. Les spécialistes en genres eux-mêmes, qui éprouvent parfois des difficultés à catégoriser, effectuent des rapprochements risqués sur la base de quelques similitudes globales perçues entre tel texte et telle catégorie, avant d'opérer des ajustements au moyen d'analyses trait par trait. Qu'ils s'en rendent compte ou non, ils utilisent ainsi des prototypes ou des visions stéréotypées. En outre, dire qu'une œuvre est un roman classique ou qualifier une pièce de théâtre de traditionelle n'est rien d'autre qu'un jugement de prototypicalité, puisque de telles affirmations découlent directement d'un appariement avec le ou un des prototypes stockés dans la mémoire. On a aussi affaire au jugement de prototypicalité chaque fois qu'on parle des «œuvres déviantes par rapport (...) aux stéréotypes » (Chiss $1987: 16$ ).

Dans le même ordre d'idées, on oppose, en poétique générique et en typologie des discours, les classifications savantes aux classifications populaires et l'on confronte « les typologies produites par les experts qui peuvent expliciter leur critères, à celle des non-experts (...)» (Chiss et Filliolet 1987 : 4). Canvat (2002), quant lui, s'interroge sur la coexistence des catégories classificatoires exogènes (savantes: type, mode) et endogènes (ordinaires) qui sont selon lui essentiellement au nombre de quatre : récit, poésie, théâtre et littérature d'idées.

La poétique générique est aussi consciente depuis longtemps de la tension qui existe entre la généricité auctoriale et lectoriale (Schaeffer 1989 : 148-156), tension génératrice de doutes et de perturbations, ainsi que le notent Chiss et Filliolet: "l'intervention des paramètres constitués par les pôles de la production et de la réception affecte aussi la pureté et la stabilité des modèles » $(1987: 6)$. Schaeffer relève ici en particulier, pour le régime lectorial, les phénomènes de rétroaction générique (1989 : 147) dont la conséquence est une «ouverture » permanente de l'identité générique du texte. Celui-ci, explique-t-il, «ne saurait prédéterminer toutes ses parentés ultérieures avec des textes ou des classes de textes encore inexistants au moment de sa production, ces parentés dépendant autant des textes à venir (et des changements historiques éventuels concernant les critères de classification) que des propriétés intrinsèques du texte en quesition » (1989:148-149). Il en va autrement du régime auctorial où la seule perspective d'analyse générique est celle de «la tradition antérieure du texte» (1989:148). Ceci dit, force est de constater que la réflexion systématique sur la variation et la stabilité interindividuelle, interculturelle et diachronique dans la manière de percevoir les genres est encore indigente ${ }^{13}$.

${ }^{13}$ Il faut pourtant rendre justice aux travaux comme Paralittérature de Boyer, où l'on trouve des observations intéressantes sur la structure du lectorat littéraire. Ces recherches sont pourtant centrées 


\section{PROTOTYPE ET ARCHÉTYPE}

Qu'on ouvre une session internet et qu'on tape dans un moteur de recherche la combinaison de mots littérature + prototype ou genre + prototype, les résultats qui s'affichent montreront que le terme de prototype n'est pas utilisé uniquement pour désigner un personnage-type. On le rencontre aussi dans des contextes plus spécifiquement génériques. Dans un article consacré au statut générique de l'essai, Renouprez écrit par exemple que «si un genre prend assise dans l'histoire littéraire, il revient à Montaigne d'avoir fondé le prototype de cette écriture et de lui avoir donné son nom " (www.orees.concordia.ca). Le terme de prototype qui se réfère ici au texte des Essais est à prendre dans le sens courant de premier exemplaire qui ouvre une série d'autres qui viendront après en en reproduisant plus ou moins fidèlement la forme générale. Kleiber précise pourtant que, pour les théoriciens du prototype, il s'agit «d'une acception technique différente du sens courant de premier exemplaire d'un modele (...)» (1990:48). A l'intérieur d'une conception restreinte de la prototypicalité, il faut donc se garder de mettre un point d'équivalence entre prototype et archétype. L'œuvre qui inaugure un genre n'en est pas nécessairement un prototype. Ce serait faire fausse route que de considérer comme prototype de texte dramatique la première pièce d'Echyle ou de tenir L'Illiade d'Homère pour le prototype du texte narratif. Le premier et le second possèdent des traits qui en font, paradoxalement, des instances quasi marginales de leurs catégories. On peut en conclure qu'une spécificité des textes littéraires tient en ce que les instances fondatrices peuvent présenter de nombreuses dissemblances d'avec les réalisations postérieures relevant du même genre. C'est l'effet, déjà signalé par Schaeffer, de rétroprojection générique. La configuration prototypique d'un genre peut en effet changer au fur et à mesure qu'une catégorie s'enrichit d'éléments nouveaux qui influent, par les innovations qu'ils apportent, sur la représentation que se font de cette catégorie les lecteurs/spectateurs. Ceci dit, il n'est point anormal que le modèle donnant naissance à une lignée soit doté d'un bon nombre de propriétés prototypiques pour la classe par lui inaugurée; rien n'empêche en théorie qu'il soit même parfaitement superposable à son image prototypique.

exclusivement sur cerlains genres de récil et sur la littérarité en général, elles ne s'intéressent pas à l'analyse comparative de la réception des genres littéraires. Dans le même contexte, on doit noter aussi l'existence de projets comme l'Observatoire National de la Lecture ( $\mathrm{ONL}$ ), organe consultatif auprès du ministère de l'Education nationale française dont l'une des initiatives consistait justement à examiner les pratiques scolaires des genres discursifs et littéraires. Sur le site internet de l'ONL, on lit: « À l'ćcole, pour les jeunes lecteurs, identifier les différents genres discursifs doit servir principalement à résoudre des problèmes de lecture, à apprécier les décalages ou les variations par rapport à un prototype, à argumenter face à une question d'interprétation ». 


\section{LES DIFFÉRENTES ORIGINES DU JUGEMENT DE REPRÉSENTATIVITÉ} CATÉGORIELLE

Lorsqu'un objet se trouve promu au rang d'instance prototypique, il peut l'être pour deux raisons : 1) parce qu'il est familier aux sujets ; 2) parce qu'il condense un grand nombre de traits dits typiques ou saillants de sa catégorie. Cette double origine du jugement de représentativité catégorielle parait être en rapport avec le problème des nivaux de catégorisation : «le critère de la familiarité s'avère pertinent pour expliquer le choix des meilleures instances des catégories superordonnées, alors que la typicalité ou possession d'attributs saillants de la catégorie prévaut plutôt pour les catégories de base " (Kleiber 1990:136). Suivant cette hypothèse, pour la super-catégorie de narratif, ou de littéraire, les interrogés choisissant évidemment le roman, le feraient parce que c'est le genre de loin le plus connu et familier. Par contre, pour trouver le prototype du roman, ils chercheraient à travers le corpus des textes connus des instances individuelles présentant des traits typiques ou, pour être plus précis correspondant au prototype du genre. Cette recherche les mènerait alors tantôt à Bazin, tantôt à Balzac, selon les compétences. Dans le cas de la généricité littéraire, il semble donc qu'en définitive, c'est le critère de familiarité qui s'avère, pour ainsi dire, dominant.

Le recours au critère de familiarité ne va pourtant pas sans difficultés. Veuillons bien admettre, à titre d'exemple, qu'à la question de savoir quel est pour eux le meilleur exemplaire de roman, des lecteurs interrogés répondent Le Père Goriot ou $A$ la recherche du temps perdu, ou qu'ils évoquent comme synonyme de pièce de théâtre, Le Mariage de Figaro ou Les Chaises. De tels choix - avouons-le - ne semblent guère improbables. Or, malgré leur incontestable notoriété et leur caractère emblématique, $A$ la recherche... et Les Chaises ne sont point des ouvrages représentatifs de leurs catégories, bien au contraire, ils s'en écartent sous maints égards. L'inexactitude consistant à appeler $A$ la recherche $d u$ temps perdu roman est comparable à celle qui consiste à ranger le dauphin dans la catégorie des poissons. Ces erreurs sont pourtant des phénomènes de prototypie par excellence.

\section{PROTOTYPE ET HYBRIDITÉ GÉNÉRIQUE}

On dit et répète que c'est la polysémie qui constitue la pierre d'achoppement de la TP. Faute de place, nous ne discuterons pas cette opinion, nous voudrions en revanche réfléchir un instant à la question de savoir s'il est fondé de parler de genres «polysémiques». Pour répondre à cette question, il est nécessaire de changer d'appareil notionnel. Le terme équivalent de polysémie, en poétique, est hybridité. Celle-ci, à l'instar de son homologue sémantique, pose de nombreux problèmes liés au statut pluricode ou inter-médias de certaines réalisations (p. ex. théâtrales) ou à l'hétérogénéité componentielle des textes (cf. Viswanathan, Grassin 2003). Si l'on 
se tient dans une perspective rigoureusement textocentriste (notanment pour le théâtre), il faut dire tout d'abord qu'il n'est pas exact de parler de genres hybrides, comme on le fait parfois, mais de textes hybrides, c'est-à-dire présentant des propriétés typiques à des genres divers. Un mode, type ou genre ne sauraient être hybrides eux-mêmes, ils peuvent seulement être plus ou moins (im)parfaitement actualisés dans les œuvres. Certains écarts sont pourtant tolérés à l'intérieur des genres les plus permissifs et génériquement vulnérables (roman, théâtre) qui invitent même aux expérimentations. Celles-ci, pratiquées de plus en plus souvent, génèrent des textes hybrides que l'on regroupe sous des appelations spéciales (p.ex. théâtroman). Une fois constitués en une sorte de catégorie autonome, ils peuvent théoriquement donner lieu à la prototypisation. En critique littéraire, on admet d'ailleurs communément que l'œuvre de M. Duras fournit un excellent exemple, c'est-dire une sorte de prototype - du théâtroman. Viswanathan et Grassin observent pourtant non sans raison qu'il s'agit là « d'une indétermination plutôt que d'un mélange " (2003). Il n'est pas ainsi tout à fait évident que l'hybridité générique puisse être traitée avec succès par la TP. Ce problème attend des propositions de théorisation.

\section{CONCLUSION}

Comme certains de nos travaux précédents, et notamment celui sur les tropes catégoriels, la présente étude n'a qu'un objectif : orienter l'attention de la poétique générique vers les résultats obtenus par les disciplines voisines (linguistique, analyse du discours, approches cognitives) en matière de catégorisation et de classification. Les théoriciens du prototype ont sérieusement réfléchi à ces problèmes et élaboré quelques conceptualisations intéressantes. Ainsi, une confrontation-adaptation s'imposait dont voici quelques conclusions.

$\mathrm{Vu}$ l'omniprésence des phénomènes de proto- et stéréo-typie et la souplesse des théories qui s'y rapportent, on doit reconnaitre tout d'abord que celles-ci ne paraissent pas incompatibles avec les théories relatives aux catégorisations et classements génériques littéraires. En effet, les genres littéraires font l'objet de prototypisation au même titre que tout autre type de référent, le mécanisme de prototypisation étant naturellement impliqué dans toute relation signe-référent.

Ainsi, au vu des assertions contenues dans les six thèses de la version standard de la sémantique du prototype (voir ci-dessous le chapitre «Prototype : évolution du concept »), le théoricien des genres peut formuler les remarques suivantes :

- Il n'est pas inexact de dire que certaines catégories génériques littéraires ont des contours incertains; il s'agit là surtout des catégories du niveau basique et superordonné dont la définition exige le recours aux différents aspects de l'acte discursif et qui autorisent une certaine hétérogénéité séquentielle, c'est-à-dire des insertions de séquences relevant d'autres genres que le genre encadrant (un sonnet, 
défini essentiellement par des règles syntaxiques ne permet pas de grands flottements, à la différence d'un roman ou d'une pièce de théâtre). Pour être encore plus précis, il faudrait dire qu'avec un consensus, les catégories génériques littéraires peuvent être définies de manière tout à fait satisfaisante par l'établissement d'un profll minimum (jeu de propriétés morphologiques et structurales objectives) déterminé selon la stratégie classique des CNS.

- Il est clair qu'il arrive que des objets littéraires soient rangés dans les catégories génériques sur la base de l'appariement global avec un prototype. Il faut souligner toutefois qu'en dehors de la communication ordinaire ceci est normalement une opération provisoire, un pis-aller ou une astuce argumentative. La recherche privilégie l'analyse trait par trait.

- La notion de ressemblance de famille semble inutile pour les recherches génériques en littérature. Elle est, à notre sens, une utopie théorique pour laquelle nous ne voyons pas d'exemple concret, lexical ou générique, qui puisse emporter notre adhésion.

En somme, les thèses 2, 3 et 5 de la TP standard étant partiellement applicables à l'univers générique littéraire, on peut dire que les catégories génériques littéraires présentent grosso modo une organisation interne prototypique. Cela ne nous empêche pas d'émettre sur le prototype générique une série d'autres observations.

- La manifestation la plus concrète de la prototypie générique dans la recherche est bien entendu l'activité définitionnelle. On a affaire, dans la poétique générique, à plusieurs types de définitions, depuis celles qui consistent à établir des profils minimum d'une catégorie (catégorie réduite à son indispensable ossature) jusqu'aux descriptions comportant, en plus des traits élémentaires, un grand nombre de propriétés plus ou moins basiques caractérisant telle ou telle classe de textes. Certains travaux proposent des solutions intermédiaires (Combe) ou prétendent ouvertement définir des prototypes sans pourtant accomplir cette tâche de manière rigoureuse (Adam). Définir le prototype d'une catégorie ne veut pas dire en donner la description la plus complète possible. Le prototype générique, comme tout autre prototype, est une instance médiane, il se réalise à mi-chemin entre le strict minimum et un maximum définitoire. En théorie, le dialogue en style direct n'est pas nécessaire pour qu'il y a roman. Or il est inconcevable que le roman prototypique soit privé de tels dialogues. De même, un texte dramatique dépourvu de didascalie liminaire détaillant le décor reste un texte dramatique, il ne saurait cependant être considéré comme conforme au prototype de sa catégorie.

- Le prototype générique a un statut quelque peu paradoxal: si l'on admet qu'il est une construction abstraite, une combinaison de traits typiques, on se heurte tout de suite à une évidence : ce modèle idéal existe bel et bien et il est incarné par plusieurs exemplaires concrets. Si en revanche on postule d'ériger en prototype une instance individuelle, on est amené à reconnaître une autre évidence : une multitude d'autres réalisations pourraient parfaitement la suppléer à ce poste ou venir l'y accompagner. Ces deux évidences, qui marquent sans doute la spécificité du champ 
littéraire, résultent de l'infinie variété des réalisations sémantico-syntaxiques pouvant actualiser un même genre.

- L'examen des hiérarchies catégorielles littéraires démontre que plus on s'élève dans l'échelle hiérarchique, plus on est confronté aux distinctions formelles, pertinentes pour tout l'univers da discours et non seulement pour le domaine littéraire. Plus on descend vers le détail, plus on est contraint de se contenter de critères thématiques et stylistiques.

- Il importe de noter que le prototype générique ne doit pas coïncider avec l'archétype, c'est-à-dire la configuration de traits présente dans l'œuvre qui inaugure un genre ou passe pour être le modèle fondateur d'une catégorie. La raison en est que le prototype est une structure dynamique qui varie dans la diachronie à travers les effets de rétroprojection générique.

- Comme toutes les disciplines qui font usage de catégorisation et de classification, la poétique générique connaît en effet des cas indiscutables et des cas marginaux, des textes génériquement purs et impurs (polyvalents). En ce sens, il n'est pas juste de parler de genres polyvalents ou « polysémiques ». En effet, si un mot peut admettre plusieurs référents, un terme générique n'en a qu'un: sa catégorie. Ceci dit, on est forcé de reconnaître l'existence de genres (roman, théâtre) particulièrement favorables à l'utilisation de procédés d'hybridation, qui tolèrent des intrusions d'autres genres et subissent des transformations internes provoquant des effets génériques compliqués. Au point de vue diachronique, on doit noter un nombre toujours croissant de formes mixtes qui préparent le public à l'admission de certains écarts. Cette mixité, lorsqu'elle atteint un certain degré, peut faire obstacle à l'approche prototypique, sans l'exclure complètement, puisque les formes hybrides peuvent théoriquement elles aussi avoir des prototypes... L'inclassable est encore une classe - comme le rappelle à juste titre Schaeffer.

\section{BIBLIOGRAPHIE}

Ada m J.-M. (1999), Linguistique textuelle. Des genres de discours aux textes, Nathan Université, Paris 2001 (1997), Textes. Types et prototypes, Nathan HER, Paris.

Adam J.-M., Heidmann U. (2004), Des genres à la généricité, L'exenple des contes (Perrault et Grimm), Langages, 153.

Amossy R., Herschberg-Pierot A. (1997), Stéréotypes et clichés. Langue, discours, société, Nathan, Paris.

Baylon Ch., Mignot X. (1995), Sémantique du langage, Nathan, Paris.

Beacco J.-C. (2004), Trois perspectives linguistiques sur la notion de genre discursif, Langages, 153.

Blanché R. (1979), Des catégories esthétiques, Vrin, Paris.

Boyer A.-M. (1992), La paralittérature, PUF, Paris, "Que sais-je ».

Branca-Rosoff S. (1999), Types, modes et genres : entre langue et discours, Langage et société, 87.

Chiss J.-L. (1987), Malaise dans la classification. Langue française, 74. 
Chiss J.-L., Filliolet J. (1987), La typologie des discours, Langue française, 74.

Combc D. (1989), „La marquise sortit à cinq heures...". Essai de définition linguistique du récit, Le français dans le monde, $57^{\circ}$ année, $\mathrm{n}^{\circ}$ 3-4.

Ducrot O., Schaeffer J.-M. (1999), Dictionnaire encyclopédique des sciences du langage, Seuil, "Points", Paris.

Genette G. (1986 (1979)), Introduction à l'architexte, in : G. Genette et al., Théorie des genres, Seuil, "Points Essais ", Paris.

Greimas A.J. (1970), Du sens, Seuil, « Poétique », Paris.

Kerbrat-Orecchioni C., Traverso V. (2004), Types d'interactions et genres de l'oral, Langages, 158.

Kleiber G. (1990), La Sémantique du prototype. Catégories et sens lexical, P.U.F., Paris.

Klink enberg J.-M. (2000), Précis de sémiotique générale, Seuil, « Points », Paris.

Macé M. (2005), Connaître et reconnaître un genre littéraire, in : Compétences, reconnaissance et pratiques génériques (resp. R. Baroni ct M. Mać́), colloque international organisé à Lausanne les 26 et 27 novembre 2004, et à Paris les 21 et 22 avril.

Mitterand H. (1996), La littérature française du XX' siècle, Nathan, Paris.

Mounin G. (1997 (1972)), La sémantique, Editions Payot \& Rivages, "Petite Bibliothèque Payot», Paris.

Schaeffer J.-M. (1989), Qu'esi-ce qu'un genre littéraire? Scuil, « Poétique », Paris.

Skibińska E. (2003), Pojęcie stereotypu w lingwistyce francuskiej, Etnolingwistyka, t. 15, UMCS, Lublin.

Viswanathan J., Grassin J.-M. (2003), Hybride, DILT (on line). 